\title{
Aging and tenosynovitis of the peroneal tendons: the cause and management
}

\begin{abstract}
An old man was presented with pain and swelling in the right ankle along the course of peroneal muscles. Blood tests were normal. X ray and MRI revealed bilateral hypertrophied peroneal tubercles, peroneal tendinopathy and extensive synovial effusion along both peroneal tendons, as well as along the flexor halluces longus muscle and in the subtalar joint. Peroneal tenosynovitis was secondary to the agerelated changes in the structure of connective tissue, and to repetitive overload exposure rather than the irritation by the hypertrophied peroneal tubercle. The patient was cured by conservative treatment.
\end{abstract}

Keywords: peroneal tenosynovitis, aging and tenosynovitis, peroneal tubercle hypertrophy
Volume 6 Issue I - 2019

\author{
Abdulameer H Dhiab Al-Nuaimi,' Kamal I \\ Shalan ${ }^{2}$ \\ 'Professor of Anatomy and consultant Orthopaedic surgeon, \\ Medical School,Al-Balqa Applied University, Jordan \\ 2Department of Radiology, The Specialty Hospital, Jordan
}

Correspondence: Abdulameer Al-Nuaimi, Department of Anatomy, College of Medicine, Al-Balqa Applied University,AlSalt, Jordan,Email abdulameerh@bau.edu.jo

Received: January 09, 2019 | Published: February 20, 2019

\section{Background}

Peroneal tendons provide stability to the ankle when it is bearing weight and protects it from sprains. They also help in turning the foot out and stabilize the arch while walking. ${ }^{1}$

Tenosynovitis is tendinitis with inflammation of the covering tendon sheath. The aging process is a common cause of this inflammation, it is usually seen at the middle-age and over; the cause is attributed to poor vascularity of the tendons. Repetitive microtrauma, chronic, repetitive overuse of the tendon, acute overload of the tendon, extreme trauma, strain, and excessive or unaccustomed exercise are also implicated..$^{2-4}$

On the lateral surface of the calcaneus, there are two bony projections, peroneal tubercle and the retrotrochlear eminence. Peroneal tubercle is located just inferior to the lateral malleolus of the ankle joint. ${ }^{5}$ The retrotrochlear eminence is present posterior to the peroneal tubercle. ${ }^{6}$

On the lateral aspect of the ankle, there are two retinacula; the superior and inferior peroneal retinacula. These retinacula are regions of localized thickening of the superficial aponeurosis that provide mechanical strength to prevent tendon bow stringing. Attachment sites of the retinacula can be easily visualized by the MRI ${ }^{7}$ the superior retinaculum is attached to the lateral malleolus, and directed downward and backward to be attached to the lateral surface of the calcaneus; it binds both the tendons of peroneus longus and brevis to the lateral side of the ankle. Downward, on the lateral surface of the calcaneus, the tendons diverge. The peroneus longus tendon runs below the peroneal tubercle, while peroneus brevis tendon runs forward above the peroneal tubercle. At that level, the tendons are held to the calcaneus by the inferior peroneal retinaculum, the latter is attached to the peroneal tubercle and to the calcaneus above and below, covering and holding both peroneal tendons. ${ }^{8}$ Proximal to the peroneal tubercle, peroneal tendons are covered within a common synovial sheath that splits at the level of the peroneal tubercle, to cover each peroneal tendon separately. Flexor hallucis longus tendon runs behind the medial malleolus. It runs deep to the flexor retinaculum, covered with its own synovial sheath. ${ }^{8}$

\section{Case presentation}

A 72 years old man was presented to the outpatient department at Al Balqa university clinic; his complaint was pain and swelling of two months duration, just below the lateral malleolus of his right ankle. The pain increased during walking and relieved at rest. The patient sustained foot instability following long walks. The patient lived on top of a hill, and use to climb up and get down the hill every day; his job needs to stand and walk for long periods.

On clinical examination, there was a fixed rounded tender swelling of 1.5 inch in diameter located anteroinferior to the right lateral malleolus. The foot was oedematous (Figure 1). There was some tenderness along the peroneal tendons. Active eversion and passive inversion of the foot were painful.

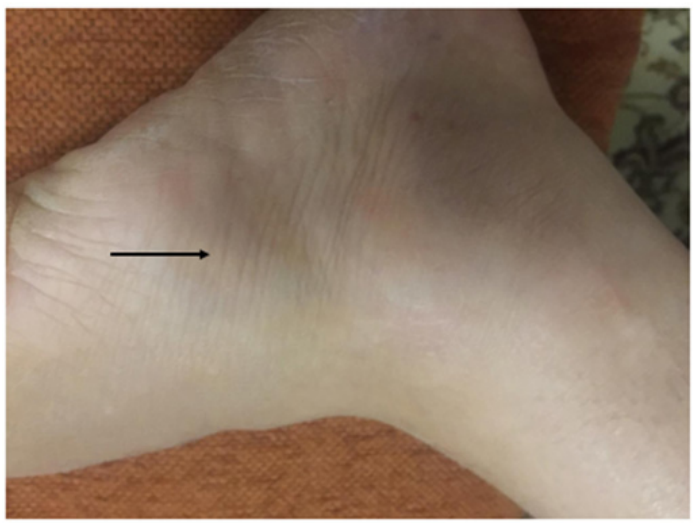

Figure I Right foot image showing fixed tender swelling anteroinferior to the lateral malleolus (black arrow) with oedematous foot.

Past history showed that the patient had an operation for right side carpal tunnel syndrome, and releasing operation for stenosing tenosynovitis of his right middle finger, four years ago. The patient has had several local corticosteroid injections for bilateral De Quervain's tenosynovitis of the extensor pollicis breves muscle.

Local stress peroneal tenosynovitis was suspected. Peroneal tenosynovitis due to chronic inflammatory disease was another alternative. Some local causes were also proposed such as, ligamentous injury of the ankle joint, torn superior peroneal retinaculum, rupture and subluxation of peroneal muscles.

\section{Investigations}

Blood tests showed normal serum levels of Uric acid, AntiStreptolysin O-Titre, C-Reactive Protein, Rheumatoid Factor, 
Vitamin-B12, and Vitamin-D3. Complete Blood Count and urine analysis were also normal.

\section{Radiography of both ankles}

Anteroposterior view of the right and left ankles showed bilateral hypertrophic peroneal tubercles on the lateral side of calcaneal bones (Figure 2).

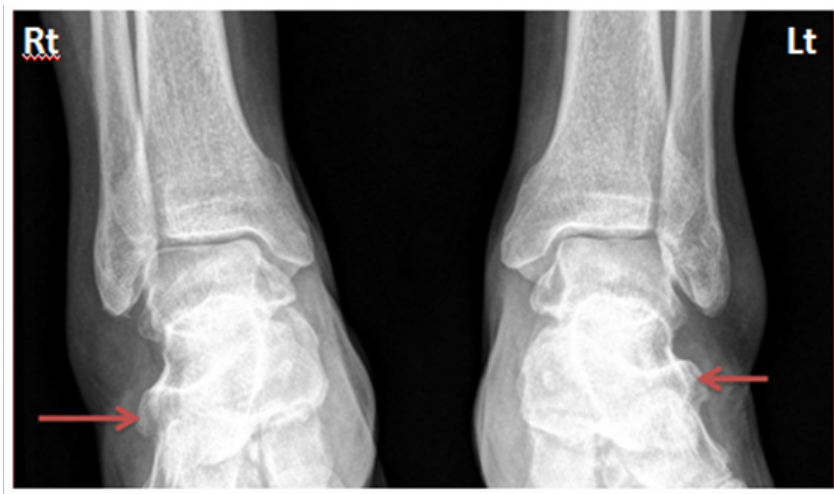

Figure 2 AP view of both right (Rt) and left (Lt) ankles revealed enlarged bilateral peroneal tubercles on the lateral side of calcaneal bones (red arrows).

\section{MRI of both ankles}

Coronal proton density MRI image of the right ankle showed a hypertrophied peroneal tubercle, it was $11.81 \mathrm{~mm}$ high, $5.52 \mathrm{~mm}$ wide, and $11.20 \mathrm{~mm}$ AP diameter. A minimal bone marrow oedema was visible in the tubercle. Both peroneal tendons were seen above the tubercle surrounded with soft tissue swelling (Figure 3).
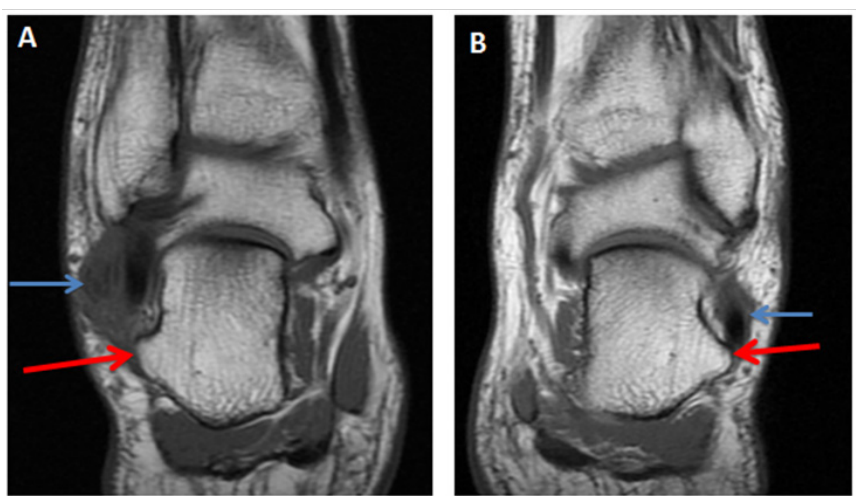

Figure 3 (A) Coronal proton density MRI image of the right ankle shows a hypertrophic peroneal tubercle (red arrow), A minimal bone marrow oedema is seen in the tubercle. Both peroneal tendons are seen above the tubercle surrounded with soft tissue swelling (blue arrow).

(B) Left side ankle for comparison shows a prominent hypertrophic peronea tubercle (red arrow). Peroneal tendons are normal (blue arrow).

The left ankle demonstrated a prominent hypertrophic peroneal tubercle; it was $9.60 \mathrm{~mm}$ high, $7 \mathrm{~mm}$ wide, and has $9.50 \mathrm{~mm} \mathrm{AP}$ diameter. Peroneal tendons were seen above the peroneal tubercle, they were normal and not surrounded with soft tissue swelling (Figure $3)$.

Coronal fat saturated proton density MRI image of the right ankle showed both peroneal tendons located above the peroneal tubercle, they were surrounded with soft tissue swelling and high T2 signal intensity which indicates fluid effusion. Abnormal signal intensity was also seen within both peroneal tendons indicating tendonitis. The left ankle showed a prominent peroneal tubercle. Peroneal tendons were normal and intact (Figure 4).
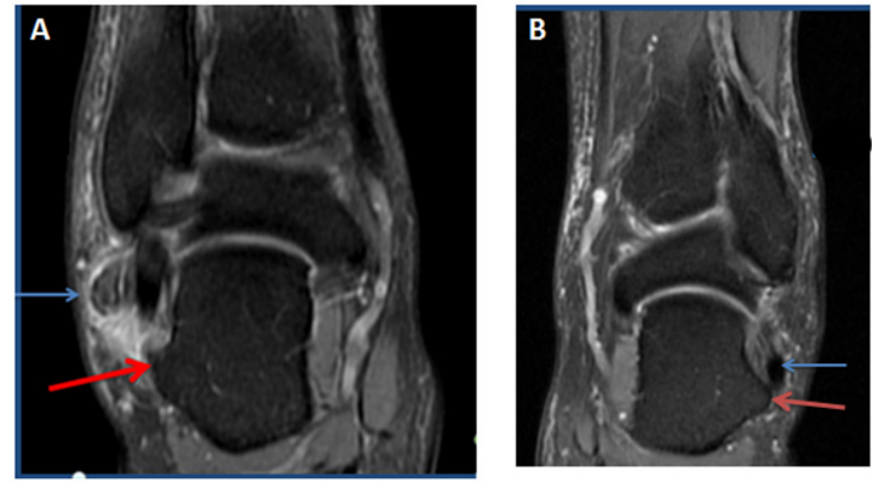

Figure 4 (A) Coronal fat saturated proton density MRI image of the right ankle shows the hypertrophic peroneal tubercle (red arrow). Both peroneal tendons are located above the tubercle, they are surrounded with soft tissue swelling and high T2 signal intensity (blue short arrow). Abnormal signal intensity is also seen within both peroneal tendons.

(B) Left ankle for comparison shows a prominent peroneal tubercle but normal peroneal tendons.

Axial fat saturated proton density MRI image of the right ankle at tibiofibular syndesmosis showed the presence of fluid within the common sheath around the peroneal tendons; it means that the fluid extension was up to the musculotendinous junction. The left side demonstrated no fluid effusion around the peroneal tendons (Figure $5 \& 6)$.
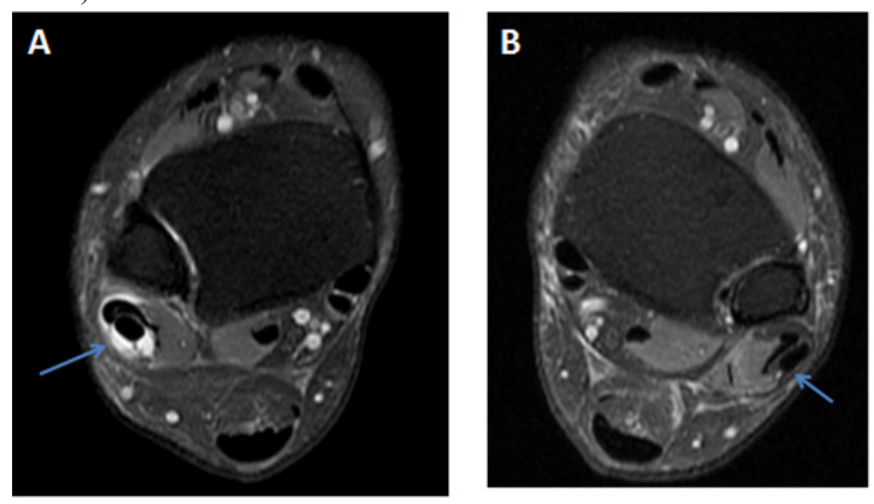

Figure 5 (A) Axial fat saturated proton density MRI image of the right ankle at tibiofibular syndesmosis shows fluid in the common sheath around the peroneal tendons (blue arrow).

(B) Left ankle for comparison shows no fluid around the peroneal tendons (blue arrow).

Sagittal fat saturated proton density MRI image of the right ankle displayed a significant amount of fluid extending down along both peroneal tendons till their bone attachments (Figure 6).

Sagittal and axial fat saturated proton density MRI images of the right ankle revealed fluid effusion in the right subtalar joint (Figures $7 \mathrm{~A} \& 7 \mathrm{~B})$

Axial fat saturated proton density MRI image of the right ankle confirmed the presence of fluid effusion around the flexor hallucis longus tendon (Figure 7C). Axial proton density MRI image of the right ankle proved intact muscular tendons and ligaments (Figure 8). 

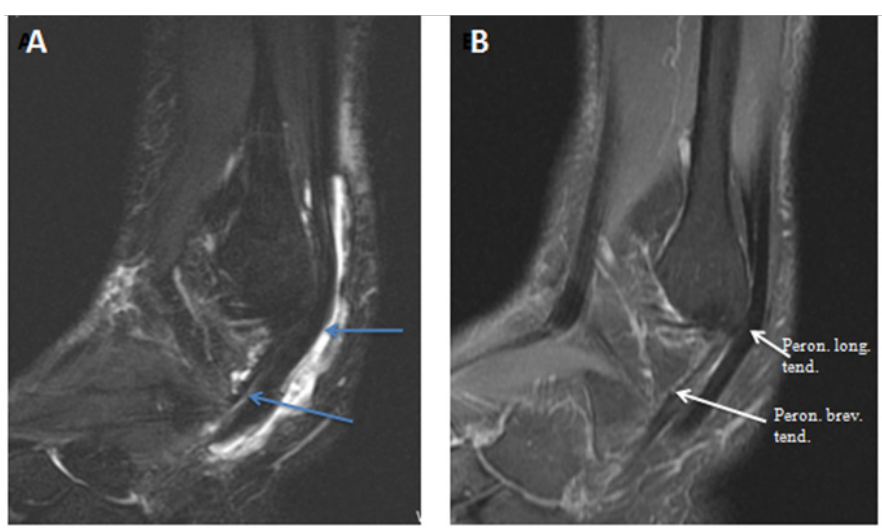

Figure 6 (A) Sagittal fat saturated proton density MRI image of the right ankle shows fluid in the common peroneal sheath and around the peroneus longus and breves tendons (blue arrows).

(B) Left ankle for comparison shows no fluid around the tendons.

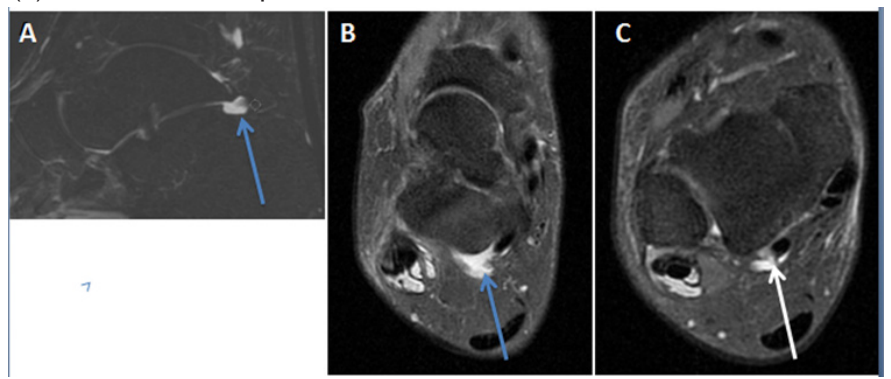

Figure 7 (A) Sagittal, (B) axial fat saturated proton density MRI images of the right ankle showing fluid in the subtalar joint (blue arrow).

(C) Axial fat saturated proton density MRI image at a lower level showing fluid around the flexor hallucis longus tendon (white arrow).

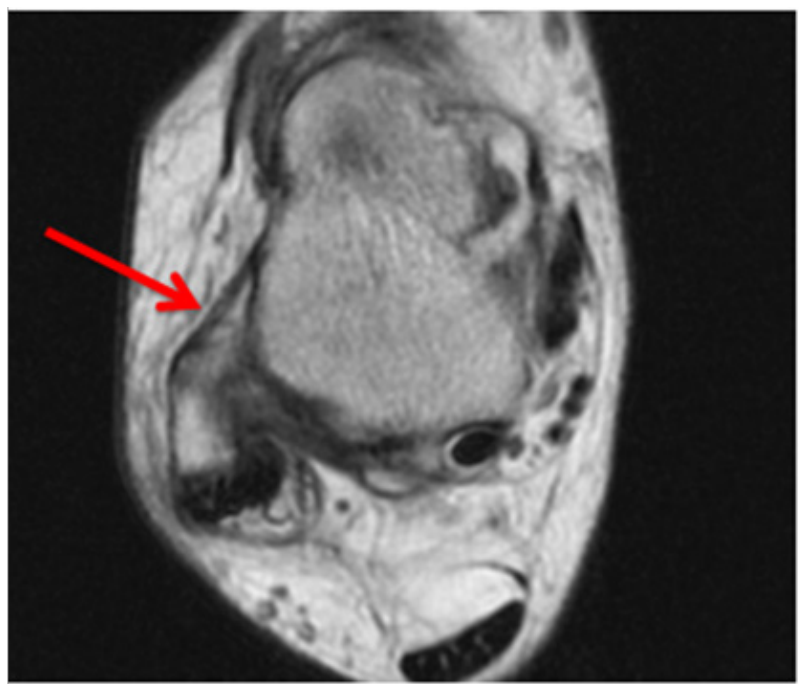

Figure 8 Axial proton density MRI image of the right ankle shows intact anterior talofibular ligament (red arrow).

\section{Management}

Complete cure was achieved within three weeks through conservative management: Rest, avoiding exercise and crepe bandage application on the ankle and foot were important. The patient was put on Nonsteroidal Anti-Inflammatory Drugs (NSAIDs).
An injection of 40mg Depo-medrol (Methylprednisolone) was given locally for successive three weeks. The drug was administered along the course of peroneal tendons.

On the fourth week following treatment, the swelling which was located anteroinferior to the lateral malleolus had disappeared and no oedema on the right foot was seen (Figure 9). The pain had disappeared, the patient was physically fit and no further management was required.

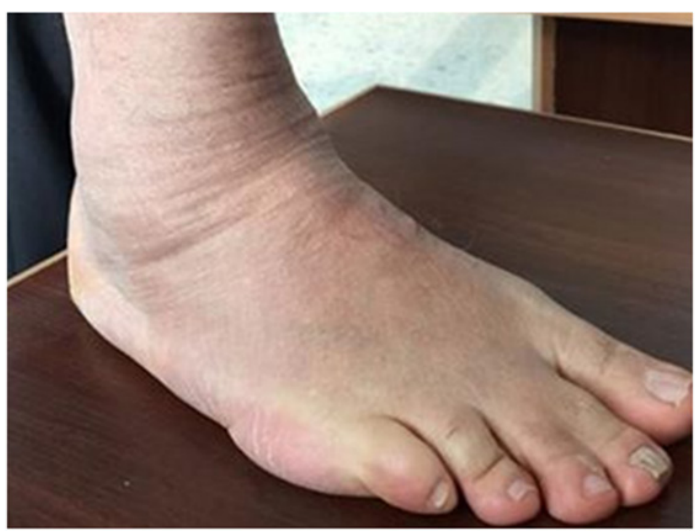

Figure 9 Right foot image taken on the fourth week following treatment. The swelling located anteroinferior to the lateral malleolus has disappeared and no oedema is seen.

\section{Discussion}

The most common causes of tenosynovitis in feet are repetitive stress activities and sudden sprains and strains. Tenosynovitis can also be due to underlying health condition such as rheumatoid arthritis, scleroderma, gout, diabetes, reactive arthritis and gonorrhoea. ${ }^{9}$

Excessive sport activities are the most likely cause for development of peroneal tenosynovitis in people having hypertrophied peroneal tubercle; tenosynovitis is attributed to the chronic irritation of the tendons and tendon sheath with the enlarged tubercle. ${ }^{10}$

Peroneal tubercle on the calcanei was identified in $90.4 \%$ of human skeletons; the length, height and width of the tubercle were 13.04, 9.44 and 3.13 respectively; the tubercle was prominent in $30 \%$ of cases. ${ }^{11}$ In another study, peroneal tubercle was considered hypertrophied when it is $5 \mathrm{~mm}$ in width. ${ }^{12}$ According to these criteria, the patient in this report had bilateral hypertrophied peroneal tubercles. Most enlarged peroneal tubercles are congenital but some are acquired; hypertrophy may be caused by trauma, abnormal weight bearing and peroneal tendonitis. ${ }^{13}$ Since the patient has bilateral hypertrophied peroneal tubercle with no evident cause, the eminent tubercles were mostly congenital.

Excessive amount of effusion was visible superiorly within the common sheath around both peroneal tendons; the fluid extends down within each splitted peroneal sheath below the level of peroneal tubercle. Peroneus brevis tendon within its sheath is located above the peroneal tubercle before reaching its destination; the movement of the tendon and the direction of muscle force are away from the tubercle. Thus, there is no reason to believe that the tenosynovitis was due to the irritation exerted by hypertrophied peroneal tubercle. This argument goes with a previous study which showed only some patients with peroneal tenosynovitis had enlarged peroneal tubercles. ${ }^{14}$ 
Inflammation of a tendon and its sheath may be caused by aging process; it is due to the poor vascularity of the tendons and connective tissue. $^{2-4}$ Other causes such as, local anatomical factors, mechanical factors, and hormonal factors are also implicated in the development of tenosynovitis. ${ }^{15}$ The patient is an old man, has had carpal tunnel release and operation on trigger finger; he also received several corticosteroid injections for tenosynovitis in both hands within the last few years. That history points out the eligibility of the patient to have some more aging inflammatory tenosynovitis.

The patient was right handedness which means right footedness. ${ }^{16}$ In right footedness people, the right leg and foot are exposed to heavier loads than the left. The residence location, long walking, and standing were reasons to over exhausting the patient's right leg muscles. The over stressing of tendons were after the development of peroneal tendinopathy (indicated on the MRI by the high signal intensity of peroneal tendons), tenosynovitis of the flexor hallucis longus muscle and subtalar effusion.

It is argued that the peroneal tenosynovitis in old people is secondary to age-related changes in the structure of connective tissue, and to repetitive overload exposure.

\section{Conclusion}

As part of senile tenosynovitis, peroneal tenosynovitis might occur in elderly people. Old people are eligible to get it following trauma or heavy exercise. Poor vascularity and degenerative changes in the connective tissue at this age are the predisposing factors. It is unwise to use a hypertrophied peroneal tubercle as a cause of this type of inflammation at that age. Conservative treatment is enough to solve the problem.

\section{Acknowledgments}

The authors are grateful to Al Balqa Applied University, Al Salt, Jordan; for the support and encouragement.

\section{Conflicts of interest}

The authors declare that they have no financial conflict of interest with regard to the content of this report.

\section{References}

1. Seymour T. What's to know about peroneal tendonitis, Medical News Today. 2018.
2. Biundo JJ. Tendinitis and tenosynovitis, Merck Manual Professional Version. 2019.

3. O'Rourke KS. Other arthropathies and regional musculoskeletal disorders. In: Hazzard WR, Blass JP, et al. editors. Principles of Geriatric Medicine and Gerontology. 5th ed. New York: McGraw-Hill; 2003:1053-1070.

4. Christian CL. Musculoskeletal syndromes. In: Barondess JA, Carpenter CCJ, editors. Differential Diagnosis. Philadelphia: Lea \& Febiger; 1994:811-884.

5. Gilcrease-Garcia B, Sambhaji CJ. et al. Radiopaedia, Peroneal tubercle. 2018.

6. Bell DJ, Sambhaji CJ, et al. Radiopaedia, retrotrochlear eminence. 2018.

7. Numkarunarunrote N, Malik A, Aguiar RO, et al. Retinacula of the foot and ankle: MRI with anatomic correlation in cadavers. AJR Am J Roentgenol. 2007;188(4):W348-W354.

8. Snell RS. Clinical anatomy. 9th ed. Philadelphia: Lippincott Williams \& Wilkins; 2011 .

9. Burke D. Tendon Sheath Inflammation (Tenosynovitis). Healthline. 2017.

10. Celikyay F, Yuksekkaya R, Almus F, et al. Tenosynovitis of the peroneal tendons associated with a hypertrophic peroneal tubercle: radiography and MRI findings. BMJ Case Rep. 2014.

11. Hyer CF, Dawson JM, Philbin TM, et al. The peroneal tubercle: description, classification, and relevance to peroneus longus tendon pathology. Foot Ankle Int. 2005;26(11):947-950.

12. Zanetti M. Founder's lecture of the ISS 2006: borderlands of normal and early pathological findings in MRI of the foot and ankle. Skeletal Radiol. 2008;37(10):875-884.

13. Boles MA, Lomasney LM, Demos TC, et al. Enlarged peroneal process with peroneus longus tendon entrapment. Skeletal Radiol. 1997;26(5)313-315.

14. Dutton P, Edmonds EW, Lark RK, et al. Prevalence of painful peroneal tubercles in the pediatric population. $J$ Foot Ankle Surg. 2012;51(5):599-603.

15. Vuillemin V, Guerini $\mathrm{H}$, Bard $\mathrm{H}$, et al. Stenosing tenosynovitis. $J$ Ultrasound. 2012;15(1):20-28.

16. Wikipedia. Laterality. 2018 